\title{
The effect of chain density on the frictional behavior of surfaces modified with alkylsiloxanes and immersed in $n$-alcohols
}

\author{
Susannah C. Clear and Paul F. Nealey a) \\ Department of Chemical Engineering, University of Wisconsin, Madison, Wisconsin 53706
}

(Received 5 May 2000; accepted 7 September 2000)

\begin{abstract}
Concepts of viscoelastic behavior of bulk polymer systems were used to describe maxima in the friction behavior of self-assembled monolayers (SAMs) measured with lateral force microscopy (LFM) as a function of sliding velocity and applied normal load in the presence of solvents (plasticizers). The objective of this study was to investigate whether decreases in the chain density of the SAMs caused maxima in the friction force to shift to higher sliding velocities; such a shift is indicative of shorter chain relaxation times in less dense, less ordered SAMs. Complete SAMs were formed from octadecyltrichlorosilane (OTS) and partial SAMs were formed from $n$-octadecylmethyldichlorosilane $(2 \mathrm{Cl})$ and $n$-octadecyldimethyldichlorosilane $(1 \mathrm{Cl})$, all on silicon/silicon dioxide substrates. With decreasing chain density, solvent partitioning into the monolayer should increase as OTS $<2 \mathrm{Cl}<1 \mathrm{Cl}$. In butanol and pentanol, the maxima in the friction force shifted to higher sliding velocities with decreasing chain density, and the relaxation times calculated for the partial $2 \mathrm{Cl}$ films were an order of magnitude shorter than those for SAMs of OTS. For both SAMs of OTS and $2 \mathrm{Cl}$ films, maxima shifted to lower sliding velocities with increases in the applied normal load and with increases in the chain length $n$ of the solvent. The higher compressibility of $2 \mathrm{Cl}$ films caused greater shifts in the maxima for similar increases in the applied normal load. The increase with $n$ was consistent with both a mechanism of solvent partitioning controlled by the free volume distribution in the SAM and a mechanism of insertion. The relaxation times of the alkyl chains were related to a molecular model of energy dissipation involving the adsorption and desorption of the chain ends to and from the surface of the probe tip. (C) 2001 American Institute of Physics.
\end{abstract}

[DOI: $10.1063 / 1.1321036]$

\section{INTRODUCTION}

Self-assembled monolayers (SAMs) of alkylsiloxanes have been identified as promising boundary lubricants for new micro- and nanoscale technologies such as microelectromechanical systems ${ }^{1-4}$ (MEMS) and fluidic selfassembly $^{5-11}$ (FSA). In liquid environments, stiction and friction forces in these applications may be reduced with appropriate selections of SAM and solvent. ${ }^{5}$ The friction behavior of SAMs is analogous to the viscoelastic behavior of bulk amorphous polymers, and in liquid environments, that behavior depends strongly on the partitioning of solvent into the SAMs. ${ }^{12-14}$ Viscoelastic theory predicts that the maximum energy dissipation and friction forces occur when the characteristic relaxation time of the monolayer is similar to the time scale associated with the sliding contact. In this paper we report maxima in the friction force as a function of sliding velocity for complete and partial SAMs of alkylsiloxanes in liquid environments. We interpret these maxima in terms of a viscoelastic model to explain how these maxima shift with changes in the chain density of the SAM and the solvent chain length.

To determine potential SAM and solvent combinations for use in FSA and MEMS devices in liquid environments, we previously used lateral force (LFM) and chemical force microscopy (CFM) to investigate the effects of terminal moi-

\footnotetext{
a) Author to whom correspondence should be addressed; electronic mail: nealey@engr.wisc.edu
}

eties and solvent environment on the adhesion and friction forces between contacting surfaces that were modified with SAMs. ${ }^{6}$ We found that SAMs of octadecyltrichlorosilane $\left(\mathrm{CH}_{3}-\left(\mathrm{CH}_{2}\right)_{17}-\mathrm{SiCl}_{3}, \mathrm{OTS}\right)$ were promising candidates for use as boundary lubricants in liquids where hydrophobic interactions were small. When SAMs of OTS were in contact with both hydrophobic and hydrophilic surfaces, they exhibited low adhesive forces and small kinetic friction coefficients in simple $n$-alcohols such as ethanol. Consequently, we selected SAMs of OTS that were immersed in $n$-alcohols, $n=1-12$, as a model system for a LFM study of the effects of sliding velocity, solvent chain length and applied normal load on the friction behavior of SAMs of alkylsiloxanes. ${ }^{12}$ Maxima in the friction force occurred as a function of sliding velocity for applied normal loads of $\sim 20-40 \mathrm{nN}$ and $n$ $=2-9$. These maxima shifted to lower sliding velocity with increasing $n$ and increasing applied normal load. These maxima were similar to maxima in the friction force reported under dry conditions for surface forces apparatus ${ }^{14}$ and LFM $^{13,15}$ studies of monolayers of amorphous dialkylammonium surfactants, as well as for LFM studies of LangmuirBlodgett films of stearic acid in the liquid expanded state ${ }^{16}$ and monolayers of unsymmetrical $n$-dialkyl sulfides on gold. ${ }^{17}$ The behavior of the maxima that we observed for SAMs of OTS in $n$-alcohols was consistent with a model of the energy dissipation in the film based on the characteristic relaxation time of the alkyl chains that is analogous to viscoelastic behavior in bulk polymer systems. The model pre- 
dicts that environmental variables that decrease the chain mobility, such as decreases in temperature, decreases in the extent of plasticization, or increases in pressure, act to increase the relaxation time and shift maxima to lower sliding velocities, and vice versa. ${ }^{14}$ In terms of this model, we interpreted these maxima as evidence of relaxation processes in the film that depend on the extent of solvent partitioning in the compressed region. ${ }^{12}$

This viscoelastic model predicts that the maxima in the friction force should shift to higher sliding velocities as the chain density of the SAMs decreases. In this paper we tested our viscoelastic model with a LFM investigation of the effect of chain density on the friction behavior of complete and partial C18 SAMs chemisorbed to silicon/silicon dioxide $\left(\mathrm{Si} / \mathrm{SiO}_{2}\right)$ surfaces as a function of the solvent environment, sliding velocity and applied normal load. We compared previously published friction data for complete SAMs of OTS ${ }^{12}$ with friction data for partial C18 SAMs formed from $n$-octadecylmethyldichlorosilane $(2 \mathrm{Cl})$ and n-octadecyldimethylchlorosilane $(1 \mathrm{Cl})$. Reported mechanisms for the deposition of tri-, di-, and monochlorosilanes suggest that the chain density decreases as $\mathrm{OTS}>2 \mathrm{Cl}>1 \mathrm{Cl}$ as the chlorine groups are replaced by methyl substituents because methyl side groups prevent the formation of interchain siloxane linkages and sterically limit the possible orientations of the head groups of the alkyl chains. ${ }^{18-20}$ We measured friction forces for surfaces modified with OTS, $2 \mathrm{Cl}$ or $1 \mathrm{Cl}$ SAMs with LFM in $n$-alcohols, $n=4-9$, at sliding velocities of $1-2000 \mu \mathrm{m} / \mathrm{s}$ and applied loads of $1-40 \mathrm{nN}$. We demonstrated shifts in the friction maxima to higher sliding velocities with decreases in the chain density, consistent with the predictions of our viscoelastic model, and interpreted friction behavior in terms of the compressibility and solvation of the SAMs.

\section{EXPERIMENT}

\section{Materials}

Polished boron-doped $\langle 100\rangle$ test grade silicon wafers were purchased from Tygh Silicon. Toluene (99.8\%, anhydrous), chloroform (99\%, anhydrous), hexadecane (99\%), and octadecyltrichlorosilane (OTS, $\left.\mathrm{CH}_{3}\left(\mathrm{CH}_{2}\right)_{17} \mathrm{SiCl}_{3}, 95 \%\right)$ were obtained from Aldrich and were used without further purification. Octadecyldimethylchlorosilane $(1 \mathrm{Cl})$ and octadectylmethyldichlorosilane $(2 \mathrm{Cl})$ were obtained from Gelest and were used without further purification. Ethanol (dehydrated, 200 proof) was obtained from Pharmco and was used as received. Water was deionized with a Millipore Academic system to a resistivity of $18.2 \mathrm{M} \Omega-\mathrm{cm}$ and a $\mathrm{pH}$ of $\sim 5.7$.

\section{$\mathrm{Si} / \mathrm{SiO}_{2}$ substrate preparation}

Wafers were cleaned by immersion in a piranha solution at $90{ }^{\circ} \mathrm{C}$ (a mixture of 7:3 (v/v) of $98 \% \mathrm{H}_{2} \mathrm{SO}_{4}$ and $30 \% \mathrm{H}_{2} \mathrm{O}_{2}$ : Caution: piranha solution reacts violently with organic compounds and should not be stored in closed containers) for $30 \mathrm{~min}$. The wafers were then removed from the piranha solution and rinsed five to six times with copious amounts of deionized water. Wafers were blown dry with nitrogen and used immediately. Freshly cleaned substrates were wet completely by deionized water. Dry wafers were transferred to a glove box filled with a nitrogen atmosphere.

\section{Deposition of alkylsiloxane films}

Complete monolayers of OTS were prepared on the freshly cleaned substrates from $0.5 \%(\mathrm{v} / \mathrm{v})$ solutions of octadecyltricholrosilane in anhydrous toluene. Kinetic studies of the thickness of the OTS film and the contact angles of water and hexadecane on OTS as functions of immersion time indicated that complete monolayers were obtained when dry $\mathrm{Si} / \mathrm{SiO}_{2}$ wafers were immersed in an unstirred solution for a period of $24 \mathrm{~h}$. Partial monolayers of $1 \mathrm{Cl}$ and $2 \mathrm{Cl}$ were prepared on freshly cleaned substrates from $1 \mathrm{mM}$ solutions of $n$-octadecyldimethylchlorosilane and n-octadecylmethyldichlorosilane in anhydrous toluene. Kinetic studies of the thicknesses of $1 \mathrm{Cl}$ and $2 \mathrm{Cl}$ films as functions of immersion time indicated that saturation coverages for these reaction conditions were obtained when dry $\mathrm{Si} / \mathrm{SiO}_{2}$ wafers were immersed in an unstirred solution for a period of $72 \mathrm{~h}$. At the end of the immersion time for each type of film, modified wafers were removed from the silanizing solution and rinsed for about $30 \mathrm{sec}$ in chloroform. Wafers then were transferred to a stirred fresh chloroform bath for $2 \mathrm{~min}$. Functionalized wafers then were removed from the nitrogen atmosphere and rinsed with absolute ethanol and deionized water and blown dry with nitrogen. Wafers modified with SAMs of OTS were transferred to a $120^{\circ} \mathrm{C}$ oven and baked for $1 \mathrm{~h} .1 \mathrm{Cl}$ and $2 \mathrm{Cl}$ films were aged at room temperature overnight prior to use. The completed OTS substrates were stored for up to six weeks in sealed petri dishes until use. The $1 \mathrm{Cl}$ and $2 \mathrm{Cl}$ films were used within four weeks.

\section{Characterization of SAMs \\ Measurement of contact angles}

The sessile contact angles, $\theta$, steady-state advancing contact angles, $\theta_{a}$, and receding contact angles, $\theta_{r}$, of deionized water and hexadecane on the SAMs formed from OTS were measured with a Ramé-Hart Model 100-00 contact angle goniometer. The methods used to measure contact angles have been described elsewhere. ${ }^{21}$ The advancing and receding contact angles of water on the SAMs of OTS of $110 \pm 2$ and $97 \pm 2^{\circ}$, as well as the advancing contact angle of hexadecane on the SAMs of OTS of $43 \pm 2^{\circ}$, agreed well with literature values. ${ }^{22-26}$ The advancing contact angles of water on $1 \mathrm{Cl}$ and $2 \mathrm{Cl}$ films at saturation coverage also were measured, and they were $102 \pm 3$ and $105 \pm 3^{\circ}$, respectively. The advancing contact angles for all the $n$-alcohols used in the friction measurements also were measured on SAMs of OTS. All the $n$-alcohols wetted the surface of the SAMs of OTS with advancing contact angles of less than $15^{\circ}$.

\section{Ellipsometry}

The thicknesses of the monolayers were measured with a Rudolf Research Type 43603-200E ellipsometer equipped with a He-Ne laser with a wavelength of $6328 \AA$ set at an incident angle of $70^{\circ}$. Measurements of the thickness of the silicon dioxide layer were made within $\sim 1 \mathrm{~h}$ of cleaning, and measurements of the alkylsiloxane surface were made within 


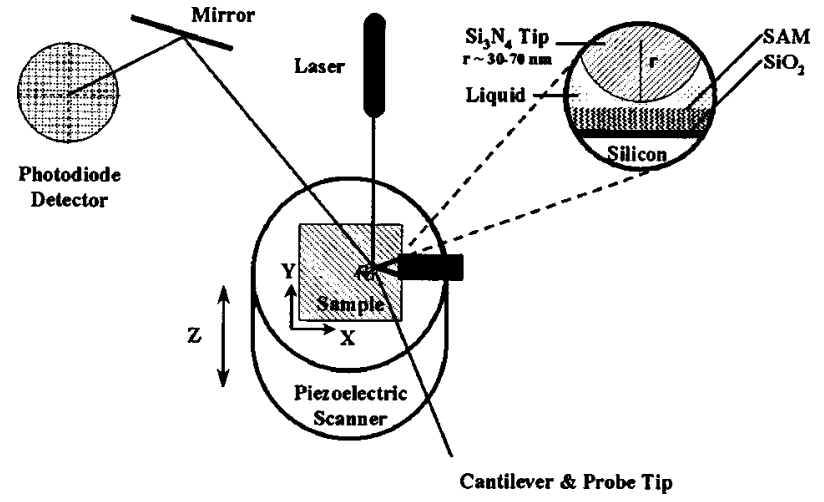

FIG. 1. Schematic of the AFM used for lateral force microscopy. The inset shows a schematic of the contacting silicon nitride tip and functionalized surface.

1 day of deposition. Because it is not possible to determine both the thickness and the index of refraction of thin films simultaneously ${ }^{27}$ the monolayer was modeled as a transparent medium with a refractive index of $1.45 .^{25,28}$ The thicknesses were determined from averages of ten separate measurements on different areas of the substrate.

\section{Scanning force microscopy}

Adhesion and friction measurements, as well as inspections of the topography of the monolayers, were done with a Nanoscope III MultiMode atomic force microscope (AFM) from Digital Instruments equipped with a fluid cell and both an E and a J scanner. $100 \mu \mathrm{m}$, wide-legged cantilevers (Digital Instruments) were used for all experiments. A schematic of the experimental setup for chemical force microscopy is shown in Fig. 1. The AFM was housed inside a sound and vibration isolation chamber. Scanning and fluid cell procedures are described elsewhere. ${ }^{12}$

Force-displacement curves were obtained at each setpoint voltage for the vertical deflection of the cantilever to determine the normal load applied to the sample. The raw voltage data was converted with Hooke's law for small cantilever deflections as described elsewhere. ${ }^{6}$ The normal spring constants for cantilevers were calibrated with a gravimetric resonance detection method as described elsewhere ${ }^{6,29}$ A value of $\sim 0.52 \mathrm{~N} / \mathrm{m}$ was calculated for cantilevers that were obtained from the same wafer. The unweighted resonant frequency of each cantilever was measured prior to use, and all cantilevers that were used had a value of approximately $60 \mathrm{kHz}$.

Friction loops were obtained for sliding velocities of $1-2000 \mu \mathrm{m} / \mathrm{s}$ over scan areas that were $500 \mathrm{~nm} \times 500 \mathrm{~nm}$ to $100 \mu \mathrm{m} \times 100 \mu \mathrm{m}$ in area with applied loads of up to $\sim 40$ nN. A representative friction loop is shown in Fig. 2. After each run, topographic images of areas that were both larger and smaller than the experimental area were obtained to check for wear. No wear was observed for any load reported in this paper. Hooke's law was applied to convert the raw voltage data to friction forces

$$
F_{\text {lateral }}=k_{\text {lat }} \Delta x=k_{\text {lat }} \frac{\Delta V}{S_{\text {lat }}},
$$

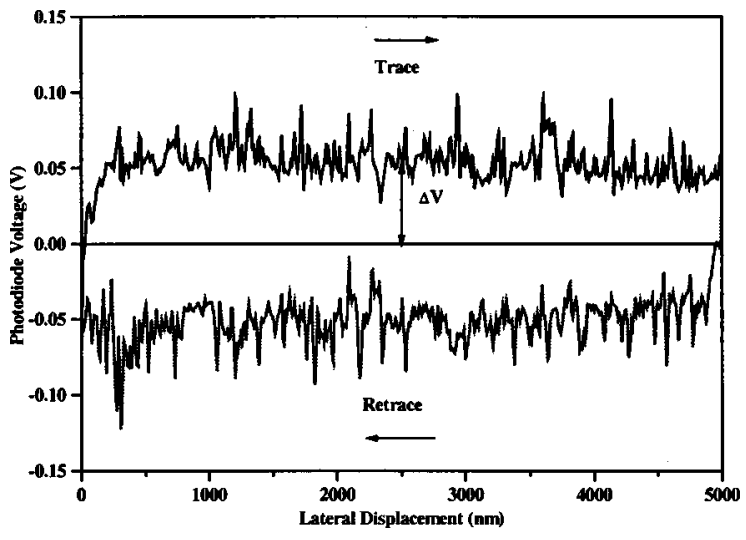

FIG. 2. Trace and retrace of a single line on a sample. The $x$-axis corresponds to the lateral deflection of the piezoelectric tube, and the $y$-axis corresponds to the torsional signal recorded by the LFM photodiode. The torsion of the cantilever is proportional to the friction force between the tip and the sample as the sample moves laterally in a direction perpendicular to the principal axis of the cantilever, and the torsion is recorded as the lateral movement of the reflected laser beam on the photodiode. This voltage signal from the photodiode may be converted into friction force with appropriate calibration of the photodiode and the cantilever.

where $k_{\text {lat }}$ is the lateral spring constant, and $S_{\text {lat }}$ is the lateral sensitivity of the photodiode. We used the method of calibration presented by Noy and co-workers to estimate the lateral spring constant, $k_{\text {lat }} \cdot{ }^{30} S_{\text {lat }}$ was obtained from friction traces over very small scan sizes, as described by Liu and co-workers ${ }^{15}$ where the torsion of the cantilever is a linear function of the lateral movement of the tip for small twisting angles. The values of the friction force reported in this paper are averages of five separate data points for each specific sliding velocity and applied normal load. Each of these individual data points was obtained from a single friction loop. The average of 512 voltages measured on the retrace was subtracted from the average of 512 voltages measured from the trace, and that difference was divided by two to determine the average LFM voltage.

\section{RESULTS AND DISCUSSION}

\section{Comparison of thicknesses of $1 \mathrm{Cl}, 2 \mathrm{Cl}$, and OTS films for qualitative estimation of chain density}

We achieved variations in the chain density by preparing alkylsiloxane films from alkylsilanes with two, one, or none of the reactive chlorine groups substituted with nonreactive methyl groups. The thicknesses of the complete and partial monolayers were measured with ellipsometry for the OTS, $2 \mathrm{Cl}$ and $1 \mathrm{Cl}$ films as a function of immersion time, as shown in Fig. 3. Complete OTS monolayers were $2.7 \pm 0.2 \mathrm{~nm}$ thick. Partial $2 \mathrm{Cl}$ and $1 \mathrm{Cl}$ monolayers were $1.9 \pm 0.2$ and 1.3 $\pm 0.2 \mathrm{~nm}$ thick, respectively. The sample area used for each measurement of the thickness was the same within the tolerances of the alignment of the ellipsometer. As a first estimate, the ratios of these measurements suggest that the surfaces modified with $2 \mathrm{Cl}$ films had $\sim 70 \%$ of the mass coverage of complete SAMs of OTS and that surfaces modified with $1 \mathrm{Cl}$ films had $\sim 40 \%$ of the mass coverage of complete SAMs of OTS. 


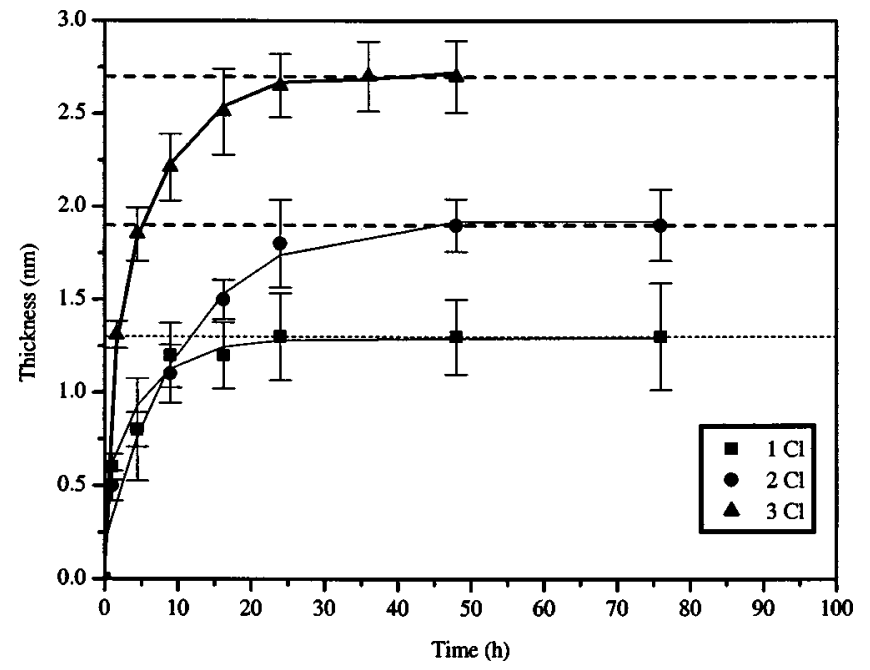

FIG. 3. Thickness measurements as a function of time for $\mathrm{C} 18$ alkylsiloxane films. (ロ) $1 \mathrm{Cl},(\bullet) 2 \mathrm{Cl},(\mathbf{\Delta})$ OTS (Ref. 12).

At saturated coverage, the chain density decreases and the free volume increases as the chlorine groups in the silanes are replaced with methyl groups because methyl groups sterically hinder the packing of the head groups of the alkylsilanes and also do not react to form covalent bonds with adjacent silane molecules or with the surface. With OTS, all of the chlorine groups can hydrolyze, permitting the formation of both surface-chain and interchain siloxane linkages. ${ }^{19,20,31-34}$ When the oxide layer has an adsorbed layer of water as it does after piranha treatment, $\mathrm{Si}-\mathrm{Cl}$ bonds may undergo hydrolysis to form a hydrogen bond with the adsorbed water layer on the surface or to react with the silanol groups on the oxide surface to form a siloxane bond. For very dilute silanizing solutions prepared from anhydrous solvents, deposition of oligomeric species from solution is negligible. The silanes adsorb by a continuous growth process initially ${ }^{33}$ to form a maximum uniform coverage with a disordered film, and then continue to adsorb to form islands of ordered regions. Interchain siloxane linkages and van der Waals forces between the long alkyl chains cause these islands to coalesce into a homogeneous ordered monolayer. ${ }^{19,31,34}$ The van der Waals forces between the chains act to orient the chains so that they are normal to the surface of the substrate. The characteristic area per chain approaches $0.21-25 \mathrm{~nm}^{2} /$ chain $^{35-37}$ for these films. $2 \mathrm{Cl}$ films also can form both surface-chain and interchain siloxane linkages, but the methyl group on each alkylsilane molecule sterically restricts interchain bonds. Consequently, there are more defect sites, and these films have greater free volume and are less ordered than SAMs of OTS. Molecules of $1 \mathrm{Cl}$ have only one group that can hydrolyze, so they chemisorb only to the silanol groups on the surface of the substrate. Rye and coworkers demonstrated with x-ray photoelectron spectroscopy that the saturation coverages of $1 \mathrm{Cl}$ films formed on hydrated silicon dioxide surfaces produced by a piranha treatment were approximately a third of the saturation coverages produced for SAMs of OTS. ${ }^{19}$ Stevens corroborated these results with a geometric simulation of the structures of $1 \mathrm{Cl}$ films that suggested that only two of the six nearest neigh- bors are allowable positions for another silane molecule. ${ }^{20}$ Both of these studies are in reasonable agreement with our estimate of $\sim 40 \%$ mass coverage for the $1 \mathrm{Cl}$ films from the thickness measurements.

\section{Friction behavior as a function of load, solvent environment, and sliding velocity}

The friction forces are plotted as functions of sliding velocity for sliding velocities of $1-2000 \mu \mathrm{m} / \mathrm{s}$ and loads of up to $\sim 40 \mathrm{nN}$ for each solvent for OTS and 2Cl SAMs in Figs. 4(a) $-4(d)$ and 5(a) $-5(d)$. Figure 6 shows data for $1 \mathrm{Cl}$ films in $n$-heptanol that are representative of the behavior of $1 \mathrm{Cl}$ films in all the solvents studied. We expect that all deformation caused by the AFM tip in these experiments was elastic because the maximum load of $\sim 40 \mathrm{nN}$ corresponds to a pressure of only $\sim 0.6 \mathrm{GPa}^{38}$

For OTS films, both inflection points followed by plateaus and maxima in the friction force appeared for $n$ $=4-9$, as shown in Figs. 4(a)-4(d). We observed at least two maxima for each solvent at applied normal loads of $\sim 20-40 \mathrm{nN}$, and these maxima shifted to lower sliding velocities by as much as $\sim 15 \mu \mathrm{m} / \mathrm{s}$ with increases in the applied normal load in this range. For $2 \mathrm{Cl}$ films, maxima in the friction force or inflection point/plateau behavior appeared for $n=4-7$. We observed two maxima in $n$-pentanol at loads of 18 and $21 \mathrm{nN}$, and an increase in the applied normal load of $3 \mathrm{nN}$ caused the maximum to shift to lower sliding velocities by nearly $600 \mu \mathrm{m} / \mathrm{s}$. For $1 \mathrm{Cl}$ films, the friction force increased in a power law fashion for all solvent and applied normal loads studied. A summary of all the velocities corresponding to the observed maxima in the friction force, or $u_{\max }$ for each applied normal load and solvent, is given in Table I. The reported maxima were determined with Table Curve two-dimensional (2D) curve-fitting software (Jandel Scientific), generally with a sum of polynomial and rational expressions for the fit of the data in the vicinity of the maximum.

In solvents where maxima or inflections in the friction force appeared, we observed three load-dependent friction regimes for both OTS SAMs and 2Cl films: low-load, intermediate-load and high-load. The low-load regime was characterized by a power law increase in the friction force with increasing sliding velocity. The intermediate-load regime corresponded to those loads where inflection points or maxima in the friction force appeared with increasing sliding velocity. In the high-load regime, the friction behavior reverted to a power law behavior, and the amount of partitioned solvent in the compressed region under the tip was negligible.

For OTS SAMs, the low-load regime behavior appeared for applied normal loads of $0-15 \mathrm{nN}$ for $n=1-9 .{ }^{12}$ The intermediate-load regime was bounded by applied normal loads of $15-40 \mathrm{nN}$ in $n$-alcohols, $n=1-9$, and maxima appeared for loads greater than $\sim 20 \mathrm{nN}$ for $n=2-9 .{ }^{12}$ The behavior consistent with the high-load regime appeared at applied normal loads greater than $\sim 40 \mathrm{nN}$. For $n$-dodecanol, the frictional behavior of OTS SAMs followed a power law for applied normal loads up to $\sim 40 \mathrm{nN}^{12}$ 


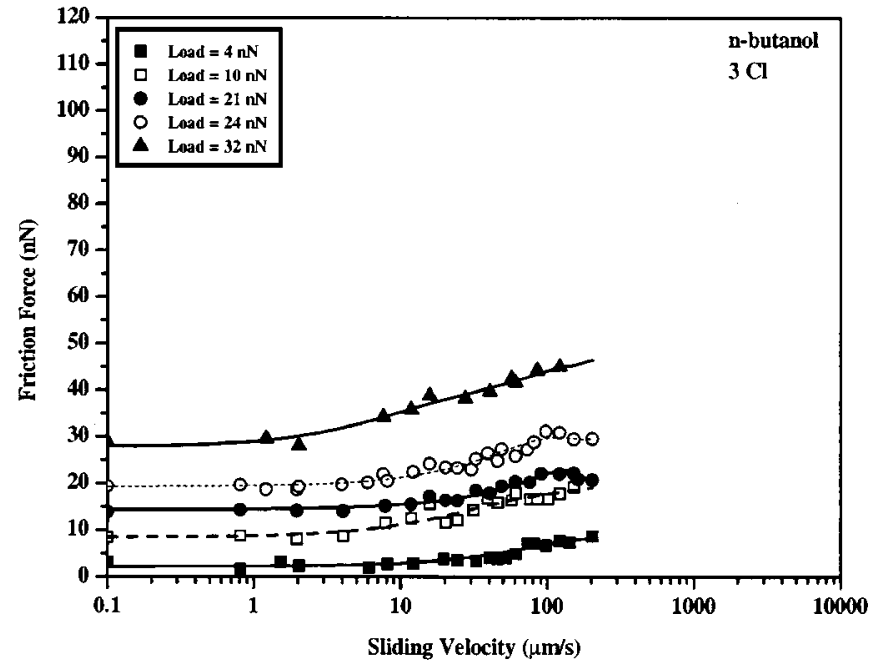

(a)

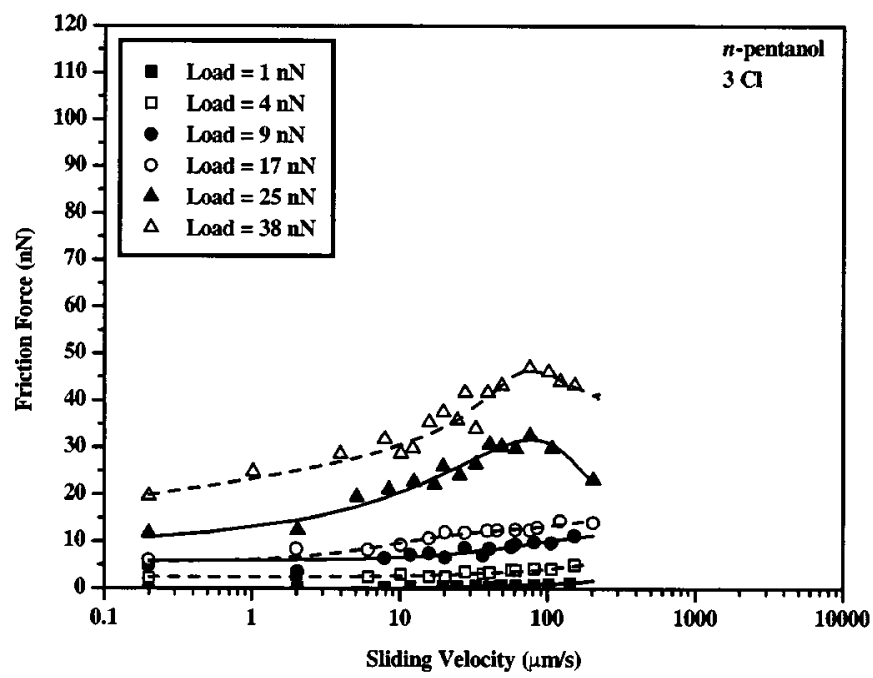

(b)

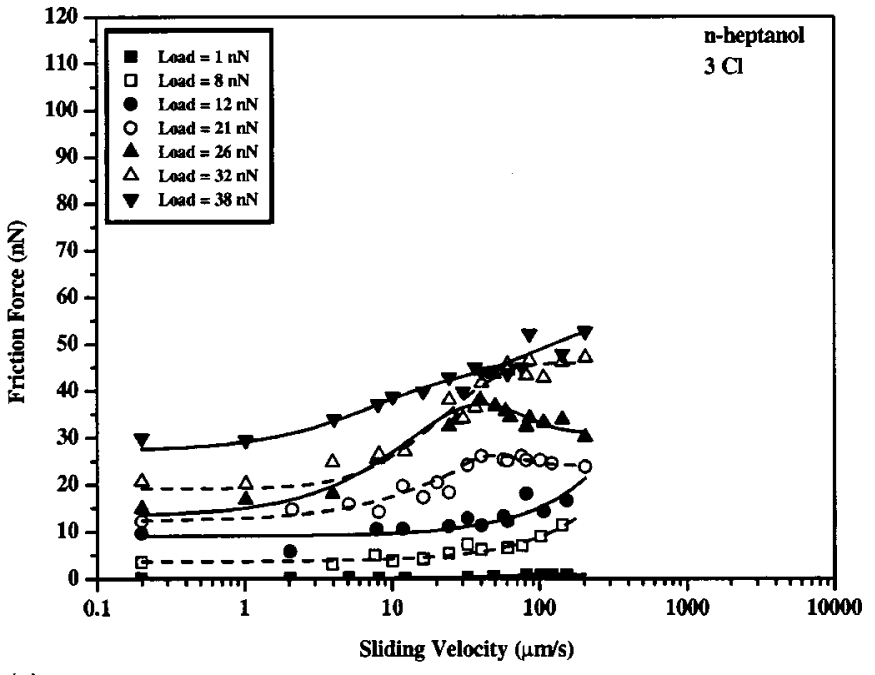

(c)

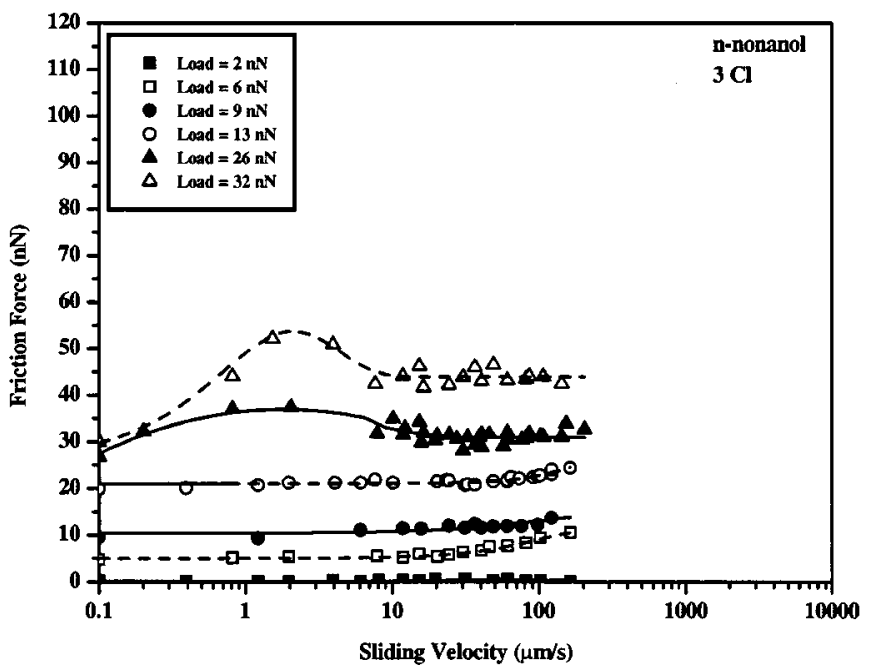

(d)

FIG. 4. Frictional forces vs sliding velocity for SAMs of OTS immersed in (a) $n$-butanol, (b) $n$-pentanol, (c) $n$-heptanol, (d) $n$-nonanol, as a function of applied normal load (Ref. 12). The lines are meant to guide the eyes.

We observed three load-dependent friction regimes for $2 \mathrm{Cl}$ films, but $1 \mathrm{Cl}$ films displayed only a power law behavior at all combinations of sliding velocity and applied normal load achieved in these experiments. For $2 \mathrm{Cl}$ films, the behavior associated with the low-load regime occurred for applied normal loads up to $10-20 \mathrm{nN}$ for a narrower range of solvent chain lengths, $n=4-7$. The boundaries of the intermediateload regime depended on the solvent environment, and we did not observe both inflection points and maxima in the same solvent. In $n$-butanol, the friction force measured on $2 \mathrm{Cl}$ films displayed a maximum for an applied normal load of $12 \mathrm{nN}$, but in $n$-heptanol, inflection points did not appear for loads less than $21 \mathrm{nN}$. The behavior associated with the high-load regime appeared at applied normal loads greater than $\sim 25 \mathrm{nN}$. Only a power law behavior appeared for applied normal loads up to $35 \mathrm{nN}$ in $n$-nonanol.

\section{Interpretation of friction maxima in terms of viscoelasticity}

As discussed elsewhere ${ }^{12}$ we interpret the appearance of maxima in the friction force in the intermediate regime as evidence of a relaxation process of alkyl chains in the complete and partial SAMs of alkylsiloxanes, analogous to relaxation processes in bulk viscoelastic systems. ${ }^{39,40}$ Viscoelastic theory for bulk systems suggests that maximum energy dissipation occurs when the characteristic relaxation time associated with conformational changes of chains is of the same order of magnitude as the time scale of the experiment. Changes in the environment or structure of a viscoelastic material that decrease the mobility of molecules in the material such as increases in load, chain order and chain density increase the characteristic relaxation time. Factors that increase the mobility of the alkyl chains such as increases in temperature or extent of plasticization, or decreases in chain order, reduce the characteristic relaxation time.

We applied viscoelastic theory to two-dimensional systems of SAMs to determine the relaxation times of the alkyl chains by defining a Deborah number, De, for the SAMs as the ratio of the characteristic relaxation time of the alkyl chains, $\tau$, to the transit time, $\tau_{t}$, or the time required for the AFM tip to move a characteristic distance, $\delta$. From a molecular viewpoint, this relaxation time may be interpreted as 

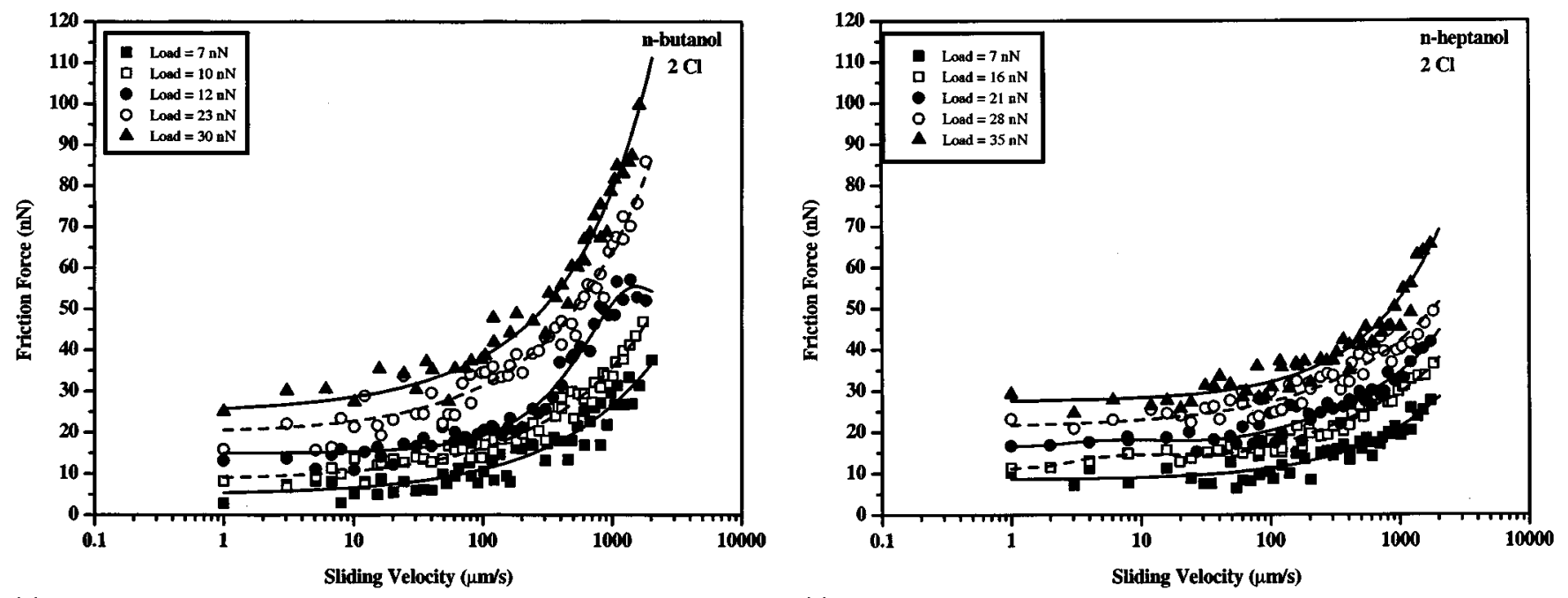

(a)

(c)
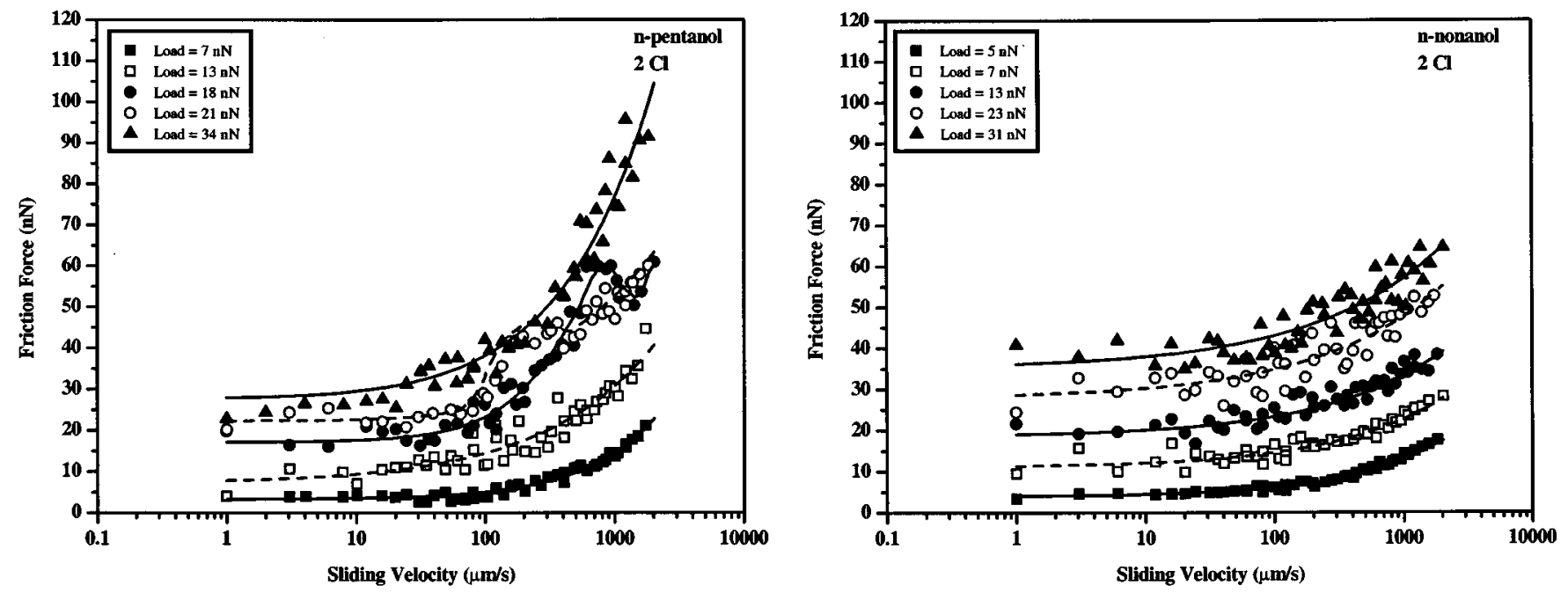

(d)

FIG. 5. Frictional forces vs sliding velocity for $2 \mathrm{Cl}$ SAMs immersed in (a) $n$-butanol, (b) $n$-pentanol, (c) $n$-heptanol, (d) $n$-nonanol, as a function of applied normal load. The lines are meant to guide the eyes.

the time that is required for a tail of an alkyl chain to adsorb to the surface of the AFM tip, elongate and tilt, and finally break free from the surface of the AFM tip. ${ }^{12}$ The transit time at a particular velocity, $u$, is given by $\tau_{t}=\delta / u$, and the viscoelastic theory presented above suggests that the maximum energy dissipation or friction force occurs when $\tau$ $\approx \tau_{t}$, or when $\mathrm{De} \approx 1$. Thus, the characteristic relaxation time is given by $\tau=\delta / u_{\max }$. Assuming $\delta$ to be equal to the chain length of the extended alkyl chains, $2.7 \mathrm{~nm}$, or the maximum distance that the alkyl chains can relax, we calculated characteristic relaxation times for alkyl chains in OTS SAMs in $n$-alcohols of $2 \times 10^{-3} \mathrm{~s}$ for $n$-nonanol and $2 \times 10^{-5} \mathrm{~s}$ for $n$-butanol. ${ }^{12}$ Alkyl chains in $2 \mathrm{Cl}$ films had characteristic relaxation times on the order of $2 \times 10^{-4} \mathrm{~s}$ in $n$-heptanol and $2 \times 10^{-6} \mathrm{~s}$ in $n$-butanol. The calculated relaxation times for the end-to-end conformational deformation of the OTS molecules in the SAM appear plausible based on a comparison to the segmental relaxation times of amorphous high molecular weight polyethylene reported by Boyd and coworkers. ${ }^{41,42}$ They reported a value of $10^{-7} \mathrm{~s}$ from molecular

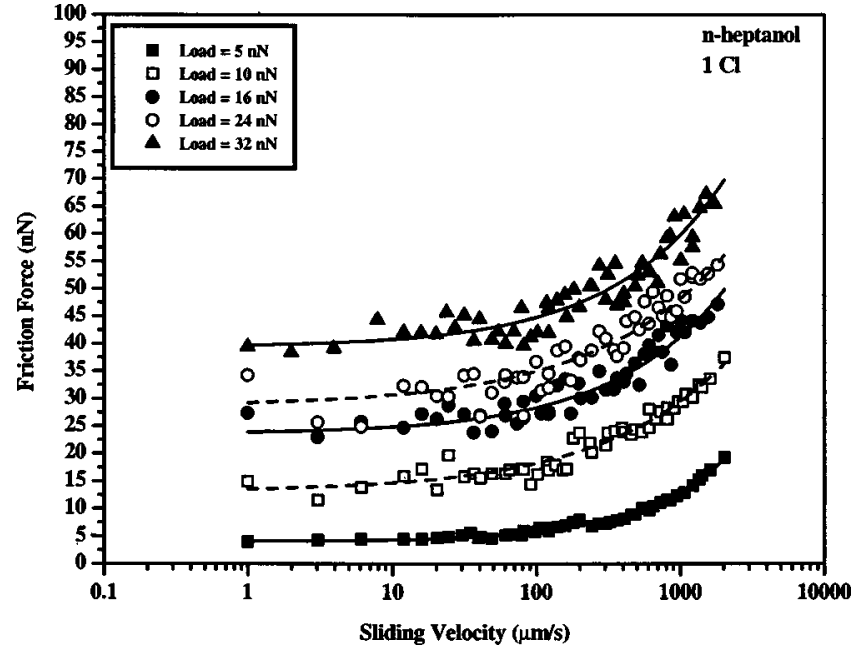

FIG. 6. Frictional forces vs sliding velocity for $1 \mathrm{Cl}$ SAMs immersed in $n$-heptanol as a function of applied normal load. The lines are meant to guide the eyes. 
TABLE I. Summary of observed maxima in the friction force in $n$-alcohols for OTS (Ref. 12) $2 \mathrm{Cl}$ and $1 \mathrm{Cl}$ SAMs.

\begin{tabular}{|c|c|c|c|c|c|c|}
\hline \multirow[b]{3}{*}{ Solvent } & \multicolumn{6}{|c|}{ Monolayer } \\
\hline & \multicolumn{2}{|c|}{$3 \mathrm{Cl}^{\mathrm{a}}$} & \multicolumn{2}{|c|}{$2 \mathrm{Cl}$} & \multicolumn{2}{|c|}{$1 \mathrm{Cl}$} \\
\hline & Load $(\mathrm{nN})$ & $u_{\max (\mu m / s)}$ & Load $(\mathrm{nN})$ & $u_{\max (\mu m / s)}$ & Load $(\mathrm{nN})$ & $u_{\max (\mu m / s)}$ \\
\hline \multirow[t]{2}{*}{$n$-butanol } & 21 & 133 & 12 & 1525 & \multicolumn{2}{|c|}{ N/A } \\
\hline & 24 & 120 & & & & \\
\hline \multirow[t]{2}{*}{$n$-pentanol } & 25 & 80 & 18 & 814 & \multicolumn{2}{|c|}{ N/A } \\
\hline & 38 & 79 & 21 & 236 & & \\
\hline \multirow[t]{2}{*}{$n$-heptanol } & 21 & 52 & $16^{\mathrm{b}}$ & $12^{\mathrm{b}}$ & \multicolumn{2}{|c|}{ N/A } \\
\hline & 26 & 41 & $21^{\mathrm{b}}$ & $8^{\mathrm{b}}$ & \multirow{3}{*}{\multicolumn{2}{|c|}{ N/A }} \\
\hline \multirow[t]{2}{*}{$n$-nonanol } & 24 & 2 & N/A & & & \\
\hline & 32 & 2 & & & & \\
\hline
\end{tabular}

${ }^{\mathrm{a}}$ Reference 12 .

${ }^{\mathrm{b}}$ Inflection point and plateau rather than a maximum.

dynamics simulations ${ }^{41}$ and a value of $\sim 10^{-6} \mathrm{~s}$ from dielectric measurements ${ }^{42}$ of the $\beta$ glass-rubber transitions of chlorine-decorated and lightly oxidized linear polyethylenes. Without taking into account plasticization effects, the relaxation times reported by Boyd and co-workers are estimated to be 2 to 3 orders of magnitude less than the calculated relaxation times of the OTS molecules in the SAM. This estimate is based on the difference between a segmental relaxation time versus the relaxation time of the end-to-end vector (longest Rouse mode) of the OTS molecules, and the difference in the relaxation times between high molecular weight chains versus chains with only 18 carbons.

This Deborah number analysis suggests that when $\tau$ increases as a result of changes in the environment or the structure of the SAM that cause the mobility of the chains to decrease, maxima in the friction force shift to lower sliding velocities, and vice versa. Decreasing the chain density should increase the mobility of the alkyl chains, causing $\tau$ to decrease and maxima to shift to higher sliding velocities, as demonstrated for OTS and $2 \mathrm{Cl}$ films by comparisons of Figs. 4(a) and 5(a) and Figs. 4(b) and 5(b). Increases in the applied normal load should reduce the mobility of the alkyl chains in the SAMs, so this analysis predicts that $\tau$ should increase and maxima should shift to lower sliding velocities, as demonstrated for OTS and 2Cl films in Figs. 4(a) $-4(d)$ and 5(a)5(c). In the low-load regime and for $1 \mathrm{Cl}$ films at all applied normal loads investigated, $\tau<\tau_{t}$, even at the highest sliding velocity investigated. In the intermediate-load regime, the appearance of maxima suggests that the values of $\tau$ were within the range of values of $\tau_{t}$ achieved. In the high-load regime, this Deborah number analysis suggests that maxima disappeared because the values of $\tau>\tau_{t}$, even at the lowest sliding velocity investigated. We only observed a decrease in $u_{\max }$ of $\sim 15 \mu \mathrm{m} / \mathrm{s}$ with similar increases in the applied normal load in the intermediate regime though, so we expect that the disappearance of maxima in the high-load regime instead may indicate that the total measured friction force is a superposition of at least the contribution associated with the relaxation process and a contribution resulting from a plowing effect. ${ }^{12}$ This contribution of plowing should increase with increased load and indentation depth, and should become dominant at high loads. This plowing contribution should have the effect of a viscous drag on the tip and should increase with sliding velocity instead of decreasing at high velocities like the contribution from the relaxation process is predicted to do. The friction data recorded at high velocities that prove the maxima to be local, such as that shown in Fig. 7 for $2 \mathrm{Cl}$ films in $n$-pentanol, support the superposition of contributions to the friction force from both plowing and the relaxation process.

We expect that the influence of increasing solvent chain length on the mobility of the alkyl chains in the monolayer and $\tau$ depends on the mechanism of solvent partitioning. As discussed elsewhere, ${ }^{12}$ solvent partitioning may be controlled by the distribution of free volume in the film $\mathrm{m}^{43}$ or partitioning may occur by insertion of long-chain solvent molecules into defect sites. ${ }^{44}$ If free-volume limited partitioning is the dominant mechanism, an increase in the extent of solvent partitioning should cause $\tau$ to decrease, just like the relaxation time and the glass transition temperature of an amorphous polymer decrease when the polymer is exposed to a plasticizer. ${ }^{45-47}$ Thus, we expect that $\tau$ will increase with increasing solvent chain length or molar volume because the extent of solvent partitioning will decrease as a result of free-volume limitations. If insertion is the dominant mechanism, solvent partitioning may cause $\tau$ to increase because

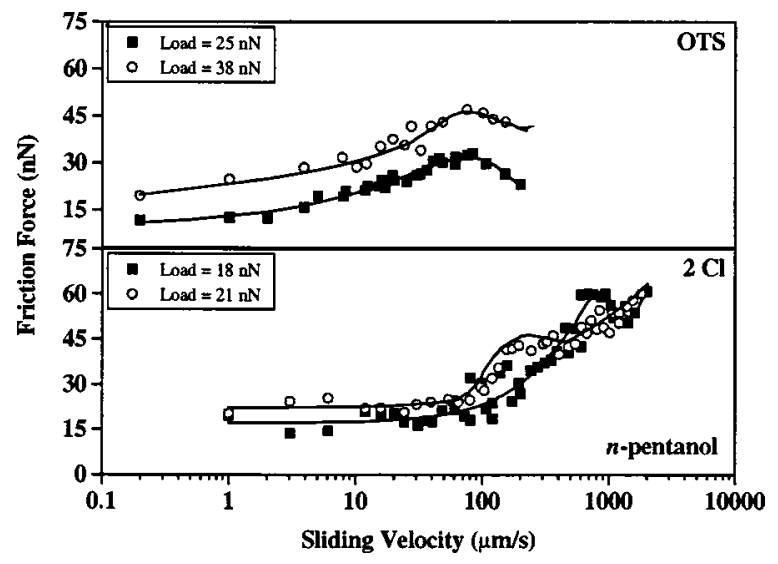

FIG. 7. Comparison of $u_{\max }$ for OTS (Ref. 12) and 2Cl SAMs in $n$-pentanol as a function of applied normal load. The lines are meant to guide the eyes. 
van der Waals forces between the alkyl chains and the inserted long-chain solvent molecules may increase the cohesive energy of the monolayer and restrict the mobility of the alkyl chains in the SAM. Theoretical calculations ${ }^{48}$ suggest that the cohesive energy increases with chain length of the solvent, so $\tau$ should increase with solvent chain length if this mechanism contributes to solvent partitioning. Both of these mechanisms of solvent partitioning are consistent with the observed shift in the maxima to lower sliding velocities with increasing solvent chain length, as demonstrated by the OTS and $2 \mathrm{Cl}$ data in Figs. 4(a)-4(d) and 5(a)-5(c).

\section{Influence of load on friction maxima changes with chain density}

The maxima in the friction force for $2 \mathrm{Cl}$ films were markedly more sensitive to changes in the applied normal load than those measured for SAMs of OTS, and we interpret this behavior in terms of the differences in compressibility of the complete and partial SAMs. Within the intermediate regime, increases in the applied normal load caused maxima to shift to lower sliding velocities for both SAMs of OTS and $2 \mathrm{Cl}$ films. A comparison of data for SAMs of OTS and $2 \mathrm{Cl}$ films in $n$-pentanol is shown in Fig. 7. In SAMs of OTS, increases in the applied normal load of 5-15 $\mathrm{nN}$ shifted $u_{\text {max }}$ to lower sliding velocities by up to $15 \mu \mathrm{m} / \mathrm{s} .{ }^{12}$ For $2 \mathrm{Cl}$ films in $n$-pentanol, a comparable increase in the applied normal load of $3 \mathrm{nN}$ caused $u_{\max }$ to decrease by $600 \mu \mathrm{m} / \mathrm{s} .2 \mathrm{Cl}$ films likely are more compressible than SAMs of OTS because they are less dense, so the same applied normal load should result in larger areas of contact and greater indentation depths for $2 \mathrm{Cl}$ films than for SAMs of OTS. Consequently, the same increase in the applied normal load should cause the reduction in mobility and the increase in $\tau$ of the alkyl chains in $2 \mathrm{Cl}$ films to be more significant than for alkyl chains in SAMs of OTS. For instance, loads of only $\sim 10-20$ $\mathrm{nN}$ are required for maxima to appear in $2 \mathrm{Cl}$ films, but SAMs of OTS must be compressed at loads of $\sim 20-25 \mathrm{nN}$ in the same solvents before maxima appear. Thus, the increased compressibility appears to be responsible for the shift in $u_{\text {max }}$ being an order of magnitude larger for $2 \mathrm{Cl}$ films than for SAMs of OTS in $n$-pentanol.

Decreases in chain density act to decrease $\tau$ even in the absence of solvent, ${ }^{16,17}$ but in liquid environments, $\tau$ and $u_{\max }$ also may depend on how the chain density or compressibility affects the extent of solvent partitioning, or plasticization, when the monolayer is compressed. A thermodynamic analysis of the solubility of the solvent in the monolayer as a function of pressure provides insight about the driving force for exclusion of solvent from the compressed region. At equilibrium, the concentration of solvent in the monolayer, $\varphi$, as a function of the uniaxial mean normal stress, $\sigma$, is given by the ratio

$$
\frac{\varphi(\sigma)}{\varphi(\sigma=0)}=\exp \left(\frac{\sigma V_{\text {penetrant }}}{R T}\right),
$$

where $\sigma=\mathrm{P} / 3, \mathrm{P}$ is the Hertzian pressure, and $V_{\text {penetrant }}$ is the molar volume of the solvent, ${ }^{4-51}$ assuming ideal mixing. The range of applied loads used in this study results in uniaxial mean normal stresses at the apex of the tip of up to $\sim 0.2 \mathrm{GPa}$. As the applied normal load increases from 0-40 $\mathrm{nN}$ for SAMs of OTS, the ratio of $\varphi / \varphi(0)$ under the apex of the tip decreases by 4 orders of magnitude for $n$-butanol and 7 orders of magnitude for $n$-nonanol. Consequently, the driving force for exclusion of solvent is significant, and $\varphi(\sigma)$ should be negligible at the highest loads investigated if the residence time or the time that an alkyl chain is compressed by the tip, $\tau_{\text {res }}$, is sufficiently long.

The actual $\varphi(\sigma)$ achieved depends on the diffusion coefficients in the SAMs and $\tau_{\text {res }}$. Both the area of contact and the thickness of the gap may affect the kinetics of the solvent exclusion. Choosing a distance of $1 \mathrm{~nm}$ as a basis, the diffusion coefficients in SAMs of OTS must be $1 \times 10^{-10} \mathrm{~cm}^{2} / \mathrm{s}$ for $n$-butanol to $2 \times 10^{-12} \mathrm{~cm}^{2} / \mathrm{s}$ for $n$-nonanol for the solvent to be displaced by that distance in $\tau_{\text {res }}$ that corresponds to the values of $u_{\max }$ at $\sim 25 \mathrm{nN} .^{12}$ Shear stresses that cause the chains to tilt forward ahead of the tip may act to reduce these diffusion coefficients further. The value for $n$-nonanol is in good agreement with the value of $1 \times 10^{-12} \mathrm{~cm}^{2} / \mathrm{s}$ that Vanderlick and co-workers reported for diffusion of pentane normal to the alkyl chains in Langmuir-Blodgett films of arachidic acid based on uptake measurements with a QCM. ${ }^{52}$ For a well-ordered film, the coefficient for lateral diffusion of the solvent is likely significantly smaller than the coefficient for diffusion normal to the alkyl chains. Consequently, we expect that for SAMs of OTS either the solvent is not excluded significantly from the compressed region under the tip or that solvent may "channel" through the SAM via a network of defects. "Channeling', that results in a larger diffusion coefficient is plausible because the molecular areas achieved in SAMS of OTS $0.21-25 \mathrm{~nm}^{2} / \mathrm{chain}^{35,36,53}$ are larger than the molecular area of the $0.19 \mathrm{~nm}^{2} /$ chain of the LB films of arachidic acid used by Vanderlick and coworkers. Also, SAMs of OTS do not display a long-range, quasicrystalline structure. $^{38}$

Both increases in load and exclusion of solvent act to increase $\tau$, and the contribution of solvent exclusion from the SAM likely becomes more important with decreasing chain density. The diffusion coefficient of an $n$-alcohol in an uncompressed $2 \mathrm{Cl}$ film may be significantly larger than in a SAM of OTS because $2 \mathrm{Cl}$ films only have $\sim 70 \%$ of the mass coverage that SAMs of OTS do. The diffusion coefficient of a particular solvent in $2 \mathrm{Cl}$ films may decrease more significantly with increases in the applied normal load than the diffusion coefficient of that same solvent in SAMs of OTS because $2 \mathrm{Cl}$ films are more compressible. Monolayers with lower chain density also should have higher values of $\varphi(0)$ than complete SAMs of OTS, and more of the alkyl chains will be solvated by the solvent molecules. At a particular uniaxial mean normal stress, $\varphi$, the difference between $\varphi(0)$ and $\varphi(\sigma)$ should be larger for $2 \mathrm{Cl}$ films than for SAMs of OTS. The applied stress should cause the alkyl chains in $2 \mathrm{Cl}$ films and other partial monolayers to undergo a transition from a plasticized, solvated state to a state where the local environment is dominated by neighboring alkyl chains as the solvent is "squeezed out" from the film. In SAMs of OTS, the solvation of the chains is minimal in comparison, so the applied stress does not cause the local 


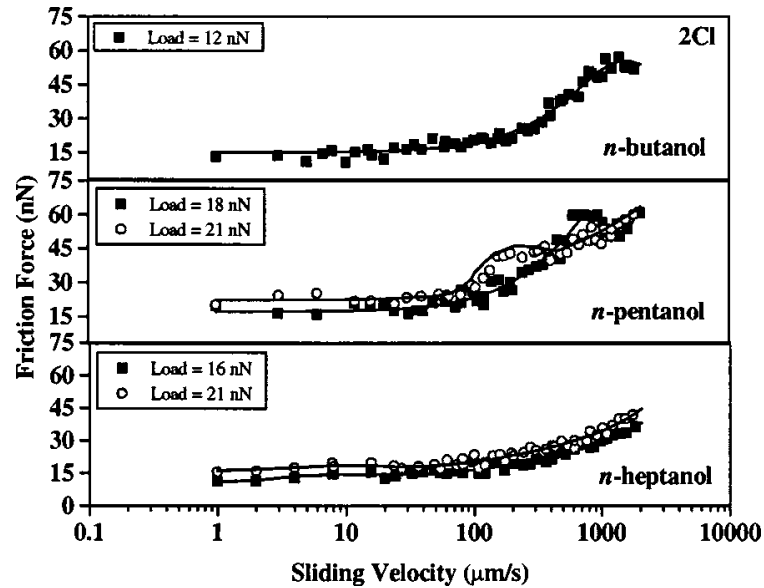

FIG. 8. Comparison of intermediate-load regime behavior for 2Cl SAMs in $n$-butanol, $n$-pentanol and $n$-nonanol. The lines are meant to guide the eyes.

solvent environment about the alkyl chains to change as dramatically as in the $2 \mathrm{Cl}$ films. Thus, $\tau$ should increase more quickly in $2 \mathrm{Cl}$ films than in SAMs of OTS, as suggested by the shifts we observed in $u_{\max }$ in Fig. 7.

\section{Effect of increasing $n$ on $u_{\max }$ for SAMs of OTS and $2 \mathrm{Cl}$ films}

The dependence of $u_{\max }$ on the chain length of the solvent was stronger for $2 \mathrm{Cl}$ films than for SAMs of OTS. We observed the intermediate-load regime for only $n=4-7$ for $2 \mathrm{Cl}$ films, rather than $n=1-9$ as we did for SAMs of OTS. ${ }^{12}$ A comparison of the intermediate-load regime behaviors recorded for $2 \mathrm{Cl}$ films is shown in Fig. 8. As shown in Fig. 8 and summarized in Table I, the value of $u_{\max }$ for $2 \mathrm{Cl}$ films decreased by an order of magnitude as $n$ increased from 4 to 5 for applied normal loads of 12 and $18 \mathrm{nN}$, respectively. In OTS films, neither a $\Delta n$ of 1 nor a $5-10 \mathrm{nN}$ shift in the applied normal load caused a shift in $u_{\text {max }}$ of this magnitude.

As the available free volume increases in the SAM, the solubility of the solvent in the SAM more strongly influences $\tau$. The solubility of the $n$-alcohols in bulk octadecane should increase with $n .^{54,55}$ Increases in solubility may lead the alkyl chains in disordered regions of the SAM to be solvated more effectively by the solvent molecules, and this should cause the repulsive forces between the chains to increase. The hard substrate only permits these repulsive forces to act laterally, effectively reducing the mobility of the solvated alkyl chains. Also, in more ordered regions of the SAM, the insertion mechanism of solvent partitioning acts to increase the cohesive energy of the monolayer as $n$ increases, or as the solvent becomes more chemically similar to the alkyl chains in the SAM. ${ }^{44,48}$ Both of these effects should cause $\tau$ to increase with $n$, and $\tau$ can span a greater range of values in $2 \mathrm{Cl}$ films than in SAMs of OTS because solvation of the alkyl chains can happen to a greater extent. This argument suggests that we only observed maxima for $n=4-7$ for $2 \mathrm{Cl}$ films because only solvation of the films by those $n$-alcohols caused the values of $\tau$ to fall within the range of transit times that corresponded to the sliding velocities we studied. For $n<4$, the repulsive forces and the increase in cohesive energy due to insertion in $2 \mathrm{Cl}$ films were likely not as significant, causing the values of $\tau$ to be shorter than the shortest transit time achieved, and vice versa for $n>7$.

\section{CONCLUSIONS}

We used LFM in a fluid cell containing a series of $n$-alcohols of chain lengths, $n=4-9$, to probe the effect of solvent partitioning, applied normal load and sliding velocity on the friction behavior of complete and partial SAMs of C18 alkylsiloxanes. We observed inflection points and maxima in the friction force for OTS and 2Cl SAMs. The values of $u_{\max }$ decreased with increasing chain density, increasing applied normal load, and increasing solvent chain length. With a Deborah number analysis analogous to those used to explain viscoelastic phenomena in bulk polymeric systems, we associated these maxima with characteristic relaxation times of the alkyl chains in these SAMs and interpreted these maxima as localized relaxation processes in the compressed region of the monolayer under the AFM tip. The shift in the values of $u_{\max }$ was inversely proportional to the change in the characteristic relaxation times. The characteristic relaxation time increased with increased applied normal load as the confinement of the alkyl chains in the SAM increased. Increases in the applied normal load also caused the relaxation time to increase because solvent was excluded from the SAM, making the monolayer less plasticized. Increases in the chain density increased the characteristic relaxation time by increasing confinement and decreasing both the compressibility and the extent of plasticization. Increases in the solvent chain length caused the relaxation time to increase as repulsive solvation forces and ordering of the alkyl chains increased.

Prior to using SAMs of alkylsiloxanes as boundary lubricants in solvent environments for applications such as FSA or MEMS, it is necessary to characterize the quality of the deposited films to determine whether the tribological properties of the SAMs are adequate for the application. Partial monolayers do not resist wear or penetration of contaminants as well as complete monolayers do. To some extent, it is possible to compensate for defect sites through use of long-chain solvents. The effectiveness and the lifespan of a device or process may be improved significantly if the range of sliding velocities achieved during operation does not include velocities near $u_{\max }$ or the high velocity range where plowing contributions at asperities become important.

\section{ACKNOWLEDGMENTS}

This research was funded by the National Science Foundation (Grant No. CTS-9703207), the National Science Foundation GOALI Program (Grant No. CTS-9520403), the Office of Naval Research DOD AASERT program, the Shell Faculty Career Initiation Fund, and the 3M Non-Tenured Faculty Award. The authors would like to acknowledge the use of facilities at the University of Wisconsin-Materials Research Science and Engineering Center for Nanostructured Materials and Interfaces (NSF). The authors also would like to acknowledge helpful discussions with S. Kim (ParkeDavis) and J. Smith (University of California-Berkeley). 
${ }^{1}$ R. Maboudian and R. T. Howe, J. Vac. Sci. Technol. B 15, 1 (1997).

${ }^{2}$ K. Deng, R. J. Collins, M. Mehregany, and C. N. Sukkenik, in Proceedings IEEE Micro-Electro-Mechanical Systems Workshop, Amsterdam, the Netherlands, 1995, pp. 368-375.

${ }^{3}$ R. L. Alley, R. T. Howe, and K. Komvopoulos, in Proc. IEEE Solid-State Sensor and Actuator Workshop, Hilton Head, SC, 1992, pp. 202-207.

${ }^{4}$ M. R. Houston, R. Maboudian, and R. T. Howe, in Proc. IEEE Solid-State Sensor and Actuator Workshop, Hilton Head, SC, 1996, pp. 42-47.

${ }^{5}$ S. C. Clear and P. F. Nealey, J. Colloid Interface Sci. 213, 238 (1999).

${ }^{6}$ H.-J. J. Yeh, Ph.D. thesis, University of California, 1994.

${ }^{7}$ H. J. Yeh and J. S. Smith, IEEE Photonics Technol. Lett. 6, 706 (1994).

${ }^{8}$ J. K. Tu, J. J. Talghader, M. A. Hadley, and J. S. Smith, Electron. Lett. 31, 1448 (1995).

${ }^{9}$ J. J. Talghader, J. K. Tu, and J. S. Smith, IEEE Photonics Technol. Lett. 7, 1321 (1995).

${ }^{10}$ A. K. Verma, M. Hadley, and J. S. Smith, in Proceedings of the 45th Electronic Components and Technology Conference '95, Las Vegas, 1995, pp. 1263-1268.

${ }^{11}$ M. A. Hadley, Ph.D. thesis, University of California, 1995.

${ }^{12}$ S. C. Clear and P. F. Nealey, Langmuir (submitted).

${ }^{13}$ Y. Liu, D. F. Evans, Q. Song, and D. W. Grainger, Langmuir 12, 1235 (1996).

${ }^{14}$ H. Yoshizawa, Y.-L. Chen, and J. Israelachvil, J. Phys. Chem. 97, 4128 (1993).

${ }^{15}$ Y. Liu, T. Wu, and D. F. Evans, Langmuir 10, 2241 (1994).

${ }^{16}$ V. V. Tsukruk, V. N. Bliznyuk, J. Hazel, D. Visser, and M. P. Everson, Langmuir 12, 4840 (1996).

${ }^{17}$ E. W. van der Vegte, A. Subbotin, G. Hadziioanou, P. R. Ashton, and J. A. Preece, Langmuir 16, 3249 (2000)

${ }^{18}$ S. Gauthier, J. P. Aime, T. Bouhacina, A. J. Attias, and B. Desbat, Langmuir 12, 5126 (1996).

${ }^{19}$ R. R. Rye, G. C. Nelson, and M. T. Dugger, Langmuir 13, 2965 (1997).

${ }^{20}$ M. J. Stevens, Langmuir 15, 2773 (1999).

${ }^{21}$ A. Ulman, An Introduction to Ultrathin Organic Films (Academic, San Diego, 1991).

${ }^{22}$ P. E. Laibinis, G. M. Whitesides, D. L. Allara, Y.-T. Tao, A. N. Parikh, and R. G. Nuzzo, J. Am. Chem. Soc. 113, 7152 (1991).

${ }^{23}$ P. E. Laibinis and G. M. Whitesides, J. Am. Chem. Soc. 114, 1990 (1992).

${ }^{24}$ R. Maoz and J. Sagiv, J. Colloid Interface Sci. 100, 465 (1984).

${ }^{25}$ S. R. Wasserman, Y.-T. Tao, and G. M. Whitesides, Langmuir 5, 1074 (1989).

${ }^{26}$ S. R. Wasserman, G. M. Whitesides, I. M. Tidswell, B. M. Ocko, P. S. Pershan, and J. D. Axe, J. Am. Chem. Soc. 111, 5852 (1989).

${ }^{27}$ F. L. McCrackin, E. Passaglia, R. R. Stromberg, and H. L. Steinberg, J. Res. Natl. Bur. Stand., Sect. A 67, 363 (1963).

${ }^{28}$ R. G. Nuzzo and D. L. Allara, J. Am. Chem. Soc. 105, 4481 (1983).
${ }^{29}$ J. P. Cleveland, S. Manne, D. Bocek, and P. K. Hansma, Rev. Sci. Instrum. 64, 403 (1993).

${ }^{30}$ A. Noy, C. D. Frisbie, L. F. Rozsnyai, M. S. Wrighton, and C. M. Lieber, J. Am. Chem. Soc. 117, 7943 (1995)

${ }^{31}$ D. L. Allara, A. N. Parikh, and F. Rondelez, Langmuir 11, 2357 (1995).

${ }^{32}$ R. R. Rye, Langmuir 13, 2588 (1997).

${ }^{33}$ T. Vallant, J. Kattner, H. Brunner, U. Mayer, and H. Hoffman, Langmuir 15, 5339 (1999).

${ }^{34}$ T. Vallant, H. Brunner, U. Mayer, H. Hoffmann, T. Leitner, R. Resch, and G. Friedbacher, J. Phys. Chem. B 102, 7190 (1998).

${ }^{35}$ K. Kojio, S. Ge, A. Takahara, and T. Kajiyama, Langmuir 14, 971 (1998).

${ }^{36}$ J. Sagiv, J. Am. Chem. Soc. 102, 92 (1980).

${ }^{37}$ I. Tidswell, B. Ocko, P. Pershan, S. Wasserman, G. Whitesides, and Axe, J. Phys. Rev. B 41, 1111 (1990).

${ }^{38}$ R. W. Carpick and M. Salmeron, Chem. Rev. 97, 1163 (1997).

${ }^{39}$ J. D. Ferry, Viscoelastic Properties of Polymers, 3rd ed. (Wiley, New York, 1980).

${ }^{40}$ G. Strobl, The Physics of Polymers (Springer-Verlag, Berlin, 1996).

${ }^{41}$ R. H. Boyd, R. H. Gee, J. Han, and Y. Jin, J. Chem. Phys. 101, 788 (1994).

${ }^{42}$ M. S. Graff and R. H. Boyd, Polymer 35, 1797 (1994).

${ }^{43}$ K. A. Dill, J. Naghizadeh, and J. A. Marqusee, Annu. Rev. Phys. Chem. 39, 425 (1988).

${ }^{44}$ K. M. R. Kallury, M. Thompson, C. P. Tripp, and M. L. Hair, Langmuir 8, 947 (1992).

${ }^{45}$ E. A. Dimarzio and J. H. Gibbs, J. Polym. Sci., Part A: Gen. Pap. 1, 1417 (1963).

${ }^{46}$ E. A. Dimarzio, C. Castellano, and A. Yang, J. Polym. Sci., Part B: Polym. Phys. 34, 535 (1996).

${ }^{47}$ B.-G. Wang, T. Yamaguchi, and S.-I. Nakao, J. Polym. Sci., Part B: Polym. Phys. 38, 846 (2000).

${ }^{48} \mathrm{~J}$. N. Israelachvili, Intermolecular and Surface Forces, 2nd ed. (Academic, New York, 1992).

${ }^{49}$ H. R. Brown, A. S. Argon, R. E. Cohen, O. S. Gebizlioglu, and E. J. Kramer, Macromolecules 22, 1002 (1989).

${ }^{50}$ H. R. Brown, J. Polym. Sci., Part B: Polym. Phys. 27, 1273 (1989).

${ }^{51}$ P. F. Nealey, R. E. Cohen, and A. S. Argon, Macromolecules 27, 4193 (1994).

${ }^{52}$ C. M. Hanley, J. A. Quinn, and T. K. Vanderlick, AIChE J. 42, 1234 (1996).

${ }^{53}$ I. M. Tidswell, T. A. Rabadeau, P. S. Pershan, and S. D. Kosowsky, J. Chem. Phys. 95, 2854 (1991).

${ }^{54}$ U. Domanska and K. Domanski, Fluid Phase Equilibria 68, 103 (1991).

${ }^{55}$ J. M. Prausnitz, R. N. Lichtenthaler, and E. G. Azevedo, Molecular Thermodynamics of Fluid-Phase Equilibria, 2nd ed. (Prentice Hall, New York, 1986). 